\title{
MISSILE CONTROL DESIGN BASED ON THE LINEAR MULTIPLE SLIDING MODE RECURSIVE METHOD
}

\author{
Y. J. SHI ${ }^{\bowtie 1}$ and G. F. MA ${ }^{2}$ \\ (Received 14 February, 2008; revised 18 June, 2008)
}

\begin{abstract}
To ensure that the elevator of a cruise missile is operating within the design specification in high-attitude flight, we present a design method for the construction of a sliding mode recursive variable structure controller. In this design method, a target sliding mode surface is first designed without considering the engineering specification of the elevator. Secondly, by using this specification, the critical state is solved. Then, the transitional sliding mode surfaces are designed recursively by using the critical state of the previous sliding mode surface so that the state will move smoothly from one transitional sliding mode surface to the next until the target sliding mode surface. This design method is based on linear sliding mode variable structure theory. Thus, the controller obtained is simple in structure and practical. Furthermore, the elevator will operate within the engineering specification. The simulation results show the effectiveness of the proposed method.
\end{abstract}

2000 Mathematics subject classification: 93B12.

Keywords and phrases: cruise missile, variable structure control, sliding mode control, multiple sliding mode recursive method.

\section{Introduction}

The sliding mode variable structure theory is an attractive tool for the construction of controllers with excellent properties, such as its low-order, robustness and ease of implementation. In the study of missile flight control, it has been widely applied in engineering [1, 4, 9-11].

In missile flight control, there are some successful applications of the sliding mode variable structure control method. In [8], novel smooth second-order sliding mode (SSOSM) control is studied. In [3], an application of adaptive control techniques is presented for the design of a skid-to-turn missile autopilot. An adaptive controller is obtained by combining the gain scheduling approach with the eigenstructure

\footnotetext{
${ }^{1}$ University of Electronic Science and Technology of China, Chengdu, P R China; e-mail: Yingjing.Shi@gmail.com.

${ }^{2}$ School of Astronautics, Harbin Institute of Technology, Harbin, P R China.

(C) Australian Mathematical Society 2008, Serial-fee code 0334-2700/08
} 
assignment control design. In [6], a sliding mode controller is derived for an integrated missile autopilot in its guidance loop. In [5], a new method is introduced to design the sliding mode control by optimally selected sliding surfaces for a class of nonlinear systems. In [12], the sliding mode control is applied to design a robust homing missile guidance law, where an adaptive reaching parameter of the sliding mode for a linear time-varying system is obtained and used to derive an adaptive sliding mode guidance law. In all of these methods, there is no mechanism to limit the large elevator deflection angle of the cruise missile which occurs in high-attitude flight.

To keep the deflection angle of the elevator within the allowable specification, the multiple sliding mode variable structure design method is proposed. The controller obtained by using this method will ensure that the missile can operate in a large flight airspace while the elevator is operating within the engineering specification. In this way, the operational performance of the missile can be improved.

The rest of the paper is organized as follows. In Section 2, we state the problem formulation of a cruise missile in high-attitude flight and describe the sliding mode controller design. In Section 3, we introduce the parameter selection principle for parameters appearing in the sliding mode control design. In Section 4, the multiple sliding surface design method is used to design a controller for a cruise missile in high-attitude flight. A simulation study is conducted in Section 5. We make some concluding remarks in Section 6.

\section{Problem formulation}

The elevator is the actuator of the cruise missile, which is installed in the tail of the missile body (for a canard missile, it is installed in the front of the body). During the flight course of the cruise missile, the moment of the missile can be produced through adjusting the angle between the elevator and airflow, and then the missile attitude is adjusted to produce the attack angle between the missile body and the airflow. As a result, a lateral force is formed and the flight trajectory is changed. The so-called overload is the ratio of the composite force of all the external forces except gravity and the missile weight, which denotes the force condition of the missile; therefore, the overload can describe the variable quantity of speed within unit time. It is a common index for maneuverability of the missile. Usually the flight course of a missile is divided into two stages. Firstly, when the missile is far from the target, since there is no feedback information from the target, we send instructions to the elevator by using the projected overload which has been prepared by the missile-borne computer, and then the real overload can track the projected overload to achieve the control of the missile-this stage is called the self-control stage. Secondly, when the missile is close to the target, the feedback information of the missile is obtained by the seeker and is adjusted by the elevator. Consequently, the missile can reach the target- this stage is called the automatic guidance stage. In this paper, only the self-control stage is considered. 
The mathematical model describing the pitch loop of an axisymmetric normal missile [7] is given by

$$
\dot{\boldsymbol{x}}=\left[\begin{array}{cc}
0 & 1 \\
-1 / T_{M}^{2} & -2 \xi_{M} / T_{M}
\end{array}\right] \boldsymbol{x}+\left[\begin{array}{c}
0 \\
v K_{M} / g T_{M}^{2}
\end{array}\right] u,
$$

where $\boldsymbol{x}=\left[x_{1}, x_{2}\right]^{\mathrm{T}}$, with the superscript $\mathrm{T}$ denoting the transpose. The first component $x_{1}$ is the difference between the longitudinal real overload $n_{y}$ and the longitudinal projected overload $n_{y}^{*}$, and the second component $x_{2}$ is the derivative of $x_{1}$. The input $u$ is the elevator angle, $T_{M}$ is the time constant of the missile, in seconds, $\xi_{M}$ is called the relative damping coefficient of the missile, $K_{M}$ is the transfer coefficient, in $1 /$ second, $v$ is the flight velocity of the missile, and $g$ is the gravity acceleration.

For system (2.1), we choose a hyperplane $S(\boldsymbol{x})=\boldsymbol{G} \boldsymbol{x}=0$, where $\boldsymbol{G}=\left[\begin{array}{ll}C_{s} & 1\end{array}\right]$, and $C_{s}$ is referred to as the sliding mode parameter. Clearly, for an arbitrary state $\overline{\boldsymbol{x}}=\left[\bar{x}_{1}, \bar{x}_{2}\right]^{\mathrm{T}}$ on the hyperplane, if $C_{s}>0$, then the state starting from $\overline{\boldsymbol{x}}$ and moving on the hyperplane will converge to the origin of the state space. On this basis, if the surface $S(\boldsymbol{x})=\boldsymbol{G} \boldsymbol{x}=0$ is the target sliding surface, we only need to find a control $\bar{u}$ such that the condition $\dot{S}(x(t))=0$ is satisfied for the state $x(t)$ starting from any state $\overline{\boldsymbol{x}}$ on the surface $S(\boldsymbol{x})=0$ under the control $\bar{u}$. To obtain such a $\bar{u}$, we recall the definition of $S(\boldsymbol{x})=\boldsymbol{G} \boldsymbol{x}=0$ and make use of (2.1) to obtain

$$
\dot{S}(\boldsymbol{x})=\boldsymbol{G} \boldsymbol{A} \boldsymbol{x}+\boldsymbol{G} \boldsymbol{B} \bar{u}=0,
$$

where $S(\boldsymbol{x})=0$. Since

$$
\boldsymbol{G} \boldsymbol{B}=\left[\begin{array}{ll}
C_{s} & 1
\end{array}\right]\left[\begin{array}{c}
0 \\
v K_{M} / g T_{M}^{2}
\end{array}\right]=\frac{v K_{M}}{g T_{M}^{2}} \neq 0,
$$

$\boldsymbol{G} \boldsymbol{B}$ is invertible. Consequently, we obtain

$$
\bar{u}=-(\boldsymbol{G B})^{-1} \boldsymbol{G} \boldsymbol{A} \boldsymbol{x},
$$

where $S(\boldsymbol{x})=0$.

Consider the case when the state of the system is outside the sliding surface, that is $S(\boldsymbol{x}) \neq 0$. In this case, we choose

$$
\dot{S}(\boldsymbol{x})=-C_{r} \operatorname{sgn}(S(\boldsymbol{x})),
$$

where $C_{r}>0$. When $S(\boldsymbol{x})>0$, we have $\dot{S}(\boldsymbol{x})=-C_{r}$, that is, $S(\boldsymbol{x})$ is decreasing with the rate determined by the parameter $C_{r}$. On the other hand, when $S(\boldsymbol{x})<0$, we have $\dot{S}(\boldsymbol{x})=C_{r}$, that is, $S(\boldsymbol{x})$ is increasing at a rate $C_{r}$. Therefore, for a state $\tilde{\boldsymbol{x}}$ such that $S(\tilde{\boldsymbol{x}}) \neq 0$, the state starting from $\tilde{\boldsymbol{x}}$ will approach the sliding surface $S(\boldsymbol{x})=0$ at a rate $C_{r}$, where $C_{r}$ is referred to as the reaching parameter.

On the basis of what has been discussed above, we see that (2.4) is a sufficient condition for the sliding mode convergence. Now, it is necessary to find a control 
$\tilde{u}$ such that (2.4) is satisfied. Suppose that (2.4) holds. Then, from the fact that $S(\boldsymbol{x})=\boldsymbol{G} \boldsymbol{x}$,

$$
\dot{S}(\boldsymbol{x})=\boldsymbol{G}(\boldsymbol{A} \boldsymbol{x}+\boldsymbol{B} \tilde{u})=-C_{r} \operatorname{sgn}(S(\boldsymbol{x})),
$$

where $S(\boldsymbol{x}) \neq 0$. Thus,

$$
\boldsymbol{G} \boldsymbol{B} \tilde{u}=-\boldsymbol{G} \boldsymbol{A} \boldsymbol{x}-C_{r} \operatorname{sgn}(S(\boldsymbol{x})),
$$

where $S(\boldsymbol{x}) \neq 0$. From (2.2), we see that $\boldsymbol{G} \boldsymbol{B}$ is invertible and hence, by (2.5), we obtain

$$
\tilde{u}=-(\boldsymbol{G} \boldsymbol{B})^{-1} \boldsymbol{G} \boldsymbol{A} \boldsymbol{x}-(\boldsymbol{G} \boldsymbol{B})^{-1} C_{r} \operatorname{sgn}(S(\boldsymbol{x})),
$$

where $S(\boldsymbol{x}) \neq 0$. This implies that during the reaching phase, when the state is moving outside the sliding surface, the control law given by (2.6) will ensure that the sufficient condition (2.4) is satisfied.

To conclude, the control law for the whole state space is given by

$$
\begin{cases}\bar{u}=-(\boldsymbol{G B})^{-1} \boldsymbol{G} \boldsymbol{A} \boldsymbol{x}, & S(\boldsymbol{x})=0, \\ \tilde{u}=-(\boldsymbol{G B})^{-1} \boldsymbol{G} \boldsymbol{A} \boldsymbol{x}-(\boldsymbol{G B})^{-1} C_{r} \operatorname{sgn}(S(\boldsymbol{x})), & S(\boldsymbol{x}) \neq 0 .\end{cases}
$$

Since $S(\boldsymbol{x})=0$ implies $\operatorname{sgn}(S(\boldsymbol{x}))=0$, the control law can be written collectively as

$$
u=-(\boldsymbol{G B})^{-1} \boldsymbol{G} \boldsymbol{A} \boldsymbol{x}-(\boldsymbol{G B})^{-1} C_{r} \operatorname{sgn}(S(\boldsymbol{x})) .
$$

Here, the first term aims to ensure that, once the state is on the sliding surface, it is maintained to be continuously on the surface. The second term is to ensure that any state outside the sliding surface will reach the sliding surface. Since $\boldsymbol{A}, \boldsymbol{B}$ are given, and $\boldsymbol{G}=\left[\begin{array}{ll}C_{s} & 1\end{array}\right]$, it follows from (2.7) that the control law is specified by $C_{s}$ and $C_{r}$.

\section{Parameter selection principle}

The missile flight under the sliding mode control can be classified into two motion phases: the sliding phase when the state is moving on the sliding surface; and the reaching phase when the state is moving outside the sliding surface.

The following theorem gives the convergence rates for both the sliding and reaching phases.

THEOREM 3.1. On the sliding phase when the state is moving on the sliding surface the convergence rate is determined by the sliding parameter $C_{s}$. On the reaching phase when the state is moving outside the sliding surface after imposing the unit overload such that

$$
S\left(x_{0}\right)=\left[\begin{array}{ll}
C_{s} & 1
\end{array}\right]\left[\begin{array}{l}
1 \\
0
\end{array}\right]=C_{s}>0,
$$

the time taken to reach the sliding surface is equal to the ratio $C_{s} / C_{r}$ of the sliding parameter $C_{s}$ and the reaching parameter $C_{r}$. 


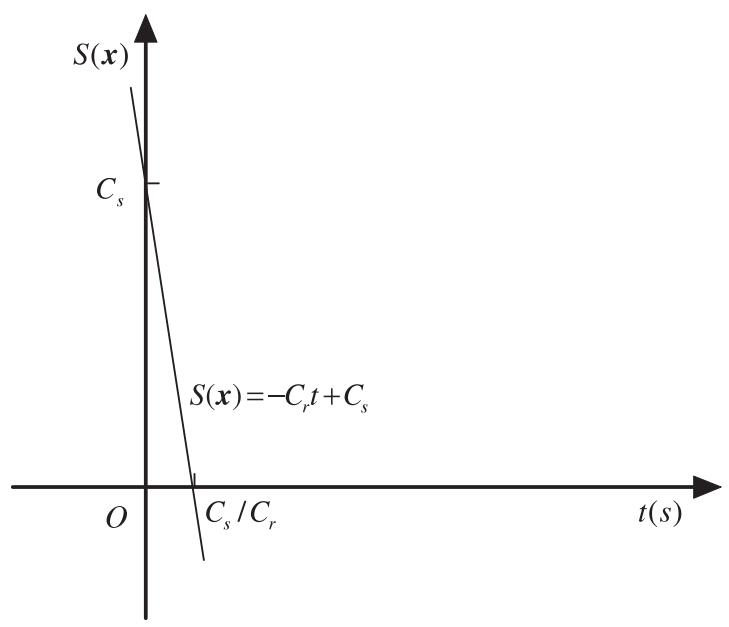

FIGURE 1. The plane diagram of $S(\boldsymbol{x})$ relative to $t$.

Proof. On the sliding phase, we have $S(\boldsymbol{x})=\boldsymbol{G} \boldsymbol{x}=0$, that is

$$
\left[\begin{array}{ll}
C_{s} & 1
\end{array}\right]\left[\begin{array}{l}
x_{1} \\
x_{2}
\end{array}\right]=0
$$

and hence

$$
C_{s} x_{1}+x_{2}=0
$$

Since $x_{2}=\dot{x}_{1}$,

$$
\dot{x}_{1}=-C_{s} x_{1}
$$

Thus,

$$
\left\{\begin{array}{l}
x_{1}(t)=\lambda e^{-C_{s} t}, \\
x_{2}(t)=-\lambda C_{s} e^{-C_{s} t},
\end{array}\right.
$$

where $\lambda \in \mathbb{R}$. Clearly, the convergence rate depends on the sliding mode parameter $C_{S}$.

On the reaching phase when the unit overload tracking is considered, the initial condition takes the form

$$
S\left(x_{0}\right)=\left[\begin{array}{ll}
C_{s} & 1
\end{array}\right]\left[\begin{array}{l}
1 \\
0
\end{array}\right]=C_{s}>0 .
$$

From (2.4), it follows that the state starting from any state $\boldsymbol{x}_{0}$ outside the sliding surface will move to the sliding surface according to $\dot{S}(\boldsymbol{x})=-C_{r}$. Figure 1 shows the plot of $S(\boldsymbol{x})$ against $t$. Clearly, with the initial condition (3.3), the time taken to converge to the sliding surface is $C_{s} / C_{r}$. This completes the proof. 
From Theorem 3.1 we know that the response speed is directly related to the sliding mode parameter $C_{s}$. That is, the larger $C_{s}$, the quicker the system response. On the other hand, if the sliding mode parameter $C_{s}$ is increased, the reaching parameter $C_{r}$ also needs to be increased, such that the system state is able to enter into the sliding surface quickly. According to (2.7), the increase of $C_{r}$ will cause difficulty in eliminating chattering. Thus, the sliding mode parameter $C_{s}$ should be chosen to be small. In this paper, the rising time for the real overload to track the unit overload is required to be less than $0.3 \mathrm{~s}$, so the sliding mode parameter is chosen as 12 . In practice, the value of the reaching parameter is usually chosen to be 10 times that of the sliding parameter.

Furthermore, the engineering specification of the elevator should also be taken into consideration. The reason for this is that the controller can only operate within the range that the elevator is able to achieve. In practice, it is known that the maximal deflection angle of the elevator occurs at the time when the system enters the sliding phase from the reaching phase. This time is called the cut-in time. Mathematically, it is defined by

$$
t_{c}=\min \{t \mid S(\boldsymbol{x}(t))=0\}
$$

The corresponding state $\boldsymbol{x}\left(t_{c}\right)$ at the cut-in time is called the cut-in point.

\section{Multiple sliding surface recursive design}

By using the single sliding mode control design method, we cannot guarantee that the deflection angle of the elevator will operate within the engineering specification. Thus, a new design method is needed. In this section, we propose to use the multiple sliding surface recursive design method.

4.1. Design process We need the following two lemmas.

LEMMA 4.1. When $S(\boldsymbol{x})>0$, the trajectory of $x_{2}$ moving outside the sliding surface is given by

$$
x_{2}(t)=\lambda e^{-C_{s} t}-\frac{C_{r}}{C_{S}},
$$

where $\lambda \in \mathbb{R}$; when $S(\boldsymbol{x})<0$, the trajectory of $x_{2}$ moving outside the sliding surface is given by

$$
x_{2}(t)=\lambda e^{-C_{s} t}+\frac{C_{r}}{C_{s}},
$$

where $\lambda \in \mathbb{R}$.

Proof. From (2.4), when $S(\boldsymbol{x})>0$, we obtain

$$
\dot{x}_{2}(t)+C_{s} x_{2}(t)=-C_{r}
$$


The general solution of (4.2) is

$$
x_{2}(t)=\lambda e^{-C_{s} t}-\frac{C_{r}}{C_{s}},
$$

where $\lambda \in \mathbb{R}$; when $S(\boldsymbol{x})<0$, we obtain

$$
\dot{x}_{2}(t)+C_{s} x_{2}(t)=C_{r}
$$

The general solution of (4.3) is

$$
x_{2}(t)=\lambda e^{-C_{s} t}+\frac{C_{r}}{C_{s}},
$$

where $\lambda \in \mathbb{R}$. This completes the proof.

LEMMA 4.2. Consider a given missile. Suppose that the target sliding surface has been constructed. Then, the elevator deflection angle at the cut-in time is proportional to the value of $x_{1}$.

PROOF. The deflection angle of the elevator is determined by (2.7), where the first term on the right-hand side of the equation is the dominating term.

Therefore, by ignoring the sign function term,

$$
u_{m}=-(\boldsymbol{G B})^{-1} \boldsymbol{G} \boldsymbol{A} \boldsymbol{x}
$$

where $\boldsymbol{A}$ and $\boldsymbol{B}$ are, respectively, the system matrix and the control matrix appeared in (2.1), and $\boldsymbol{G}=\left[\begin{array}{ll}C_{s} & 1\end{array}\right]$. Substituting the specifications of $\boldsymbol{A}, \boldsymbol{B}$, and $\boldsymbol{G}$ into (4.4), we obtain

$$
u_{m}=-\frac{g T_{M}^{2}}{v K_{M}}\left[\begin{array}{ll}
C_{s} & 1
\end{array}\right]\left[\begin{array}{cc}
0 & 1 \\
-1 / T_{M}^{2} & -2 \xi_{M} / T_{M}
\end{array}\right]\left[\begin{array}{l}
x_{1} \\
x_{2}
\end{array}\right]
$$

Since the state satisfies (3.1) and hence (3.2) at the cut-in time,

$$
u_{m}=\frac{g T_{M}^{2}}{v K_{M}}\left(\frac{1}{T_{M}^{2}}-2 \frac{\xi_{M} C_{s}}{T_{M}}+C_{s}^{2}\right) x_{1} .
$$

Clearly, when $C_{s}$ is given, $u_{m}$ is proportional to $x_{1}$. This completes the proof.

The following theorem presents a foundation for the multiple sliding surface recursive design.

THEOREM 4.3. For any two points on the same sliding surface, the point, which is closer to the equivalent point, that isthe origin of the state space, needs a smaller deflection angle of the elevator to reach the cut-in point of the next sliding surface. 
PROOF. Let there be two sliding surfaces given by

$$
S^{0}(\boldsymbol{x})=\left[\begin{array}{ll}
C_{s}^{0} & 1
\end{array}\right]\left[\begin{array}{l}
x_{1}^{0} \\
x_{2}^{0}
\end{array}\right]=0
$$

and

$$
S^{f}(\boldsymbol{x})=\left[\begin{array}{ll}
C_{s}^{f} & 1
\end{array}\right]\left[\begin{array}{l}
x_{1}^{f} \\
x_{2}^{f}
\end{array}\right]=0 .
$$

Let $\boldsymbol{x}_{1}^{0}=\left[x_{1,1}^{0}, \quad x_{1,2}^{0}\right]^{\mathrm{T}}$ and $\boldsymbol{x}_{2}^{0}=\left[x_{2,1}^{0}, x_{2,2}^{0}\right]^{\mathrm{T}}$ be two points on the sliding surface $S^{0}(\boldsymbol{x})=0$, where $C_{s}^{0}<C_{s}^{f}$. If $x_{1,1}^{0}<x_{2,1}^{0}$, then the distances between the sliding surface $S^{f}(\boldsymbol{x})=0$ and these two points are given by

$$
S^{f}\left(\boldsymbol{x}_{i}^{0}\right)=\left[\begin{array}{ll}
C_{s}^{f} & 1
\end{array}\right]\left[\begin{array}{c}
x_{i, 1}^{0} \\
-C_{s}^{0} x_{i, 1}^{0}
\end{array}\right]=\left(C_{s}^{f}-C_{s}^{0}\right) x_{i, 1}^{0}, \quad i=1,2,
$$

respectively. The state starting from the initial states $\boldsymbol{x}_{i}^{0}, i=1,2$, outside the sliding surface $S^{f}(\boldsymbol{x})=0$ will move to the sliding surface according to $\dot{S}(\boldsymbol{x})=-C_{r}$. Thus, with the initial conditions (4.8), it is clear that the times taken to reach the sliding surface $S^{f}(\boldsymbol{x})=0$ are given by

$$
t_{i}=\frac{C_{s}^{f}-C_{s}^{0}}{C_{r}} x_{i, 1}^{0}, \quad i=1,2,
$$

respectively, where $t_{i}, i=1,2$, are the corresponding cut-in times.

By Lemma 4.1, we see that the trajectories of $x_{i, 2}$, where $i=1,2$, are

$$
x_{i, 2}=\lambda_{i} e^{-C_{s}^{f} t}-\frac{C_{r}}{C_{s}^{f}}, \quad i=1,2 .
$$

Furthermore, they both start from the states on the sliding surface $S^{0}(\boldsymbol{x})=0$. Substituting (4.9) into the sliding surface Equation (4.6),

$$
\lambda_{i}=\frac{C_{r}}{C_{s}^{f}}-C_{s}^{0} x_{i, 1}^{0}, \quad i=1,2 .
$$

Since the trajectories of $x_{i, 2}$, where $i=1,2$, at the cut-in times $t_{i}, i=1,2$, are on the sliding surface $S^{f}(\boldsymbol{x})=0$, it follows from (4.7) and (4.9) that

$$
C_{s}^{f} x_{i, 1}^{f}=\frac{C_{r}}{C_{s}^{f}}-\lambda_{i} e^{-C_{s}^{f} t_{i}}, \quad i=1,2 .
$$

Since $t_{1}<t_{2}$

$$
\frac{C_{r}}{C_{s}^{f}}-\lambda_{1} e^{-C_{s}^{f} t_{1}}<\frac{C_{r}}{C_{S}^{f}}-\lambda_{1} e^{-C_{s}^{f} t_{2}}
$$


Furthermore, $\lambda_{1}>\lambda_{2}$. Thus,

$$
\frac{C_{r}}{C_{S}^{f}}-\lambda_{1} e^{-C_{s}^{f} t_{2}}<\frac{C_{r}}{C_{S}^{f}}-\lambda_{2} e^{-C_{s}^{f} t_{2}} .
$$

Combining (4.10) and (4.11), it follows from Lemma 4.2 that the deflection angle of the elevator at the cut-in time of the state starting from $x_{1}^{0}$ is smaller than that of the state starting from $\boldsymbol{x}_{2}^{0}$. This completes the proof.

From Theorem 4.3, we see that to obtain a larger flight airspace under the condition that deflection angle of the elevator does not exceed the engineering specification, we first design the target sliding surface without taking into consideration the engineering specification on the deflection angle of the elevator. Then, by virtue of this specification, we design recursively several transitional sliding surfaces so that the state starting from outside the target sliding surface will move from one transitional sliding surface to the next until the target sliding surface is reached. The details of the design procedure are given below.

Step 1. Design the target sliding surface by using the method developed in Section 2.

Step 2. Investigate whether or not the deflection angle of the elevator exceeds the engineering design specification when the state approaches the target sliding surface. If it does not exceed the design specification, the design process is complete. If it exceeds the design specification, we solve for the critical state, which is defined by

$$
\left\{\boldsymbol{x}(t) \mid(\boldsymbol{G B})^{-1} \boldsymbol{G} \boldsymbol{A} \boldsymbol{x}(t)=\bar{\delta}\right\}
$$

where $\bar{\delta}$ is the maximum allowable deflection angle of the elevator. Note that $\boldsymbol{G B}$ is invertible.

Step 3. Design a sliding surface such that it passes through the critical state obtained in Step 2. Then, the state will first move to this new sliding surface and then move on to the target sliding surface.

Step 4. If the deflection angle of the elevator does not exceed the design specification when the state moves from the new sliding surface to the target sliding surface, then the multiple sliding surface design process is complete. If the deflection angle of the elevator exceeds the design specification, then repeat Step 2 until it satisfies the design specification.

Let there be $M$ transitional sliding surfaces constructed in Step 2 and Step 3. For each $i=1,2, \ldots, M, C_{s}^{i}$ denotes the sliding surface parameter corresponding to the $i$ th transitional sliding surface. Consider the initial sliding surface

$$
\left[\begin{array}{ll}
C_{s}^{0} & 1
\end{array}\right]\left[\begin{array}{l}
x_{1} \\
x_{2}
\end{array}\right]=0 .
$$

With the imposing of the unit overload change, the initial condition is

$$
\boldsymbol{x}^{0}=\left[\begin{array}{l}
1 \\
0
\end{array}\right] \text {. }
$$


From (4.12) and (4.13), it is clear that the sliding surface parameter $C_{s}^{0}=0$. On this basis, it follows from (4.1) and (4.5) that the sliding surface parameters $C_{s}^{i}, i=1,2, \ldots, M$, can be obtained through solving the following algebraic equations recursively:

$$
\left\{\begin{array}{l}
\bar{\delta}=\frac{g T_{M}^{2}}{v K_{M}}\left(\frac{1}{T_{M}^{2}}-2 \frac{\xi_{M} C_{s}^{i}}{T_{M}}+\left(C_{s}^{i}\right)^{2}\right) x_{i, 1}, \\
-C_{s}^{i} x_{i, 1}=\left(\frac{C_{r}}{C_{s}^{i}}-C_{s}^{i-1} x_{i-1,1}\right) \exp \left(\frac{C_{s}^{i}\left(C_{s}^{i-1}-C_{s}^{i}\right) x_{i-1,1}}{C_{r}}\right)-\frac{C_{r}}{C_{s}^{i}},
\end{array}\right.
$$

where $\bar{\delta}$ is the maximum allowable deflection angle of the elevator, and $C_{s}^{i-1}$ denotes the sliding mode parameter of the previous sliding surface.

The multiple sliding mode recursive principle may be summarized as follows. First, from the requirement of the rising time, we determine the sliding mode parameter $C_{s}$. Then, we calculate the elevator state at the cut-in point from which we decide whether a transitional sliding surface needs to be introduced. If the deflection angle of the elevator does not exceed the limited value, we do not need a transitional sliding surface. However, if the deflection angle of the elevator exceeds the limited value, a transitional sliding surface needs to be constructed. To introduce the required sliding surface, the transitional sliding parameter $C_{s}^{i}$ is obtained through solving (4.14).

4.2. Chattering elimination and stability analysis The sign function term is introduced to the sliding mode control so as to achieve stability and parameter perturbation invariance. However, the sign function also gives rise to chattering of the system, which is a potential threat to the elevator, causing resonance in the system. The elevator deflection angle curve without the elimination is shown in Figure 2. Thus, it is clear that chattering should be eliminated. In practice, this can be done by using either the saturation function method or the dead zone method. In this paper, the saturation function method is used. The main idea is to smooth out the sign function. That is, the sign function is approximated by a saturation function in some small region. Clearly, the saturation function is continuous.

More specifically, we introduce a small quantity $\delta, \delta>0$. We use the idea reported in [2] to approximate the sign function as

$$
\operatorname{sgn}\left(S_{i}(\boldsymbol{x})\right)=\frac{S_{i}(\boldsymbol{x})}{\left|S_{i}(\boldsymbol{x})\right|+\delta},
$$

where $\delta$ is called the chattering elimination parameter. The modified control law is given by

$$
\left\{\begin{array}{l}
\bar{u}_{i}=-(\boldsymbol{G} \boldsymbol{B})^{-1} \boldsymbol{G} \boldsymbol{A} \boldsymbol{x}, \quad S(\boldsymbol{x})=0, \\
\tilde{u}_{i}=-(\boldsymbol{G} \boldsymbol{B})^{-1} \boldsymbol{G} \boldsymbol{A} \boldsymbol{x}-(\boldsymbol{G} \boldsymbol{B})^{-1} C_{r} \frac{S_{i}(\boldsymbol{x})}{\left|S_{i}(\boldsymbol{x})\right|+\delta}, \quad S(\boldsymbol{x}) \neq 0 .
\end{array}\right.
$$




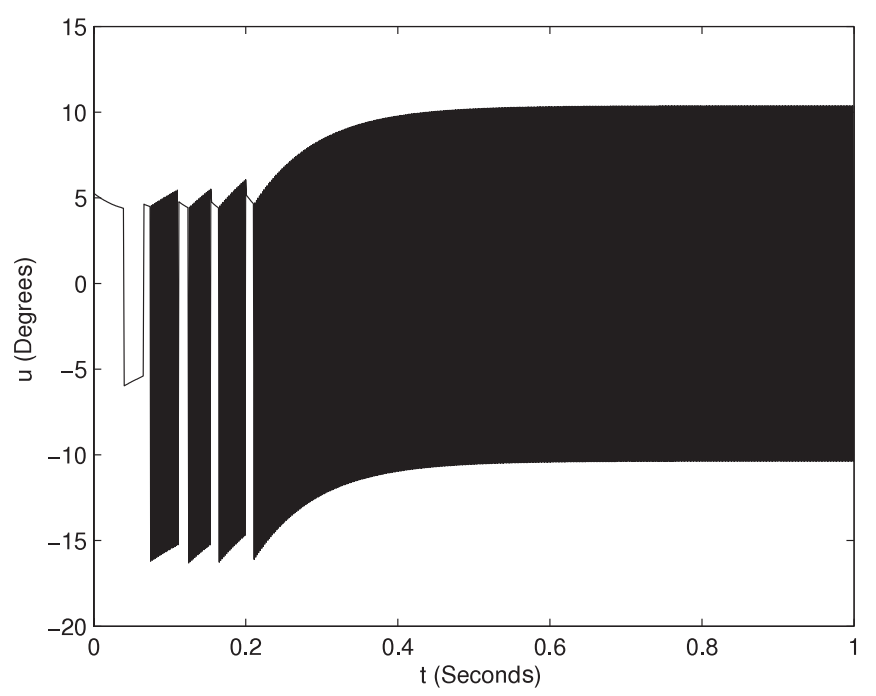

FIGURE 2. The deflection angle without elimination of chattering.

The next theorem shows the stability analysis of the multiple sliding surface control law designed by the method developed in Section 4.1.

THEOREM 4.4. The multiple sliding surface control law, designed by the design procedure detailed in Section 4.1, is a stabilizing controller for the system.

PROOF. First, consider the stability of the system when the state is moving on the sliding mode phase. Substituting the control law (2.3) into system (2.1), we obtain

$\dot{\boldsymbol{x}}=\left(\left[\begin{array}{ll}1 & 0 \\ 0 & 1\end{array}\right]+\left[\begin{array}{c}0 \\ v K_{M} / g T_{M}^{2}\end{array}\right]\left(v K_{M} / g T_{M}^{2}\right)^{-1}\left[\begin{array}{ll}C_{s}^{i} & 1\end{array}\right]\right)\left[\begin{array}{cc}0 & 1 \\ -1 / T_{M}^{2} & -2 \xi_{M} / T_{M}\end{array}\right] \boldsymbol{x}$,

where $S_{i}(\boldsymbol{x})=0$. Simplifying and arranging (4.15), we obtain

$$
\dot{\boldsymbol{x}}=\left[\begin{array}{cc}
0 & 1 \\
0 & -C_{s}^{i}
\end{array}\right] \boldsymbol{x},
$$

where $S_{i}(\boldsymbol{x})=0$. Clearly, if the sliding mode parameter $C_{s}^{i}$ is greater than zero, the system is asymptotically stable. We now consider the reaching mode phase when the state is moving outside the sliding surface. Define the Lyapunov function

$$
V(\boldsymbol{x})=\frac{1}{2} S(\boldsymbol{x})^{\mathrm{T}} S(\boldsymbol{x}),
$$

where $S(\boldsymbol{x}) \neq 0$, while $S(\boldsymbol{x})=0$ is the target sliding surface. Since $S(\boldsymbol{x}) \neq 0$ on the reaching phase, $V(\boldsymbol{x})>0$. In addition, since the designed control law satisfies (2.4), we obtain

$$
\dot{V}(\boldsymbol{x})=-C_{r} \operatorname{sgn}(S(\boldsymbol{x})) S(\boldsymbol{x})^{\mathrm{T}}<0,
$$




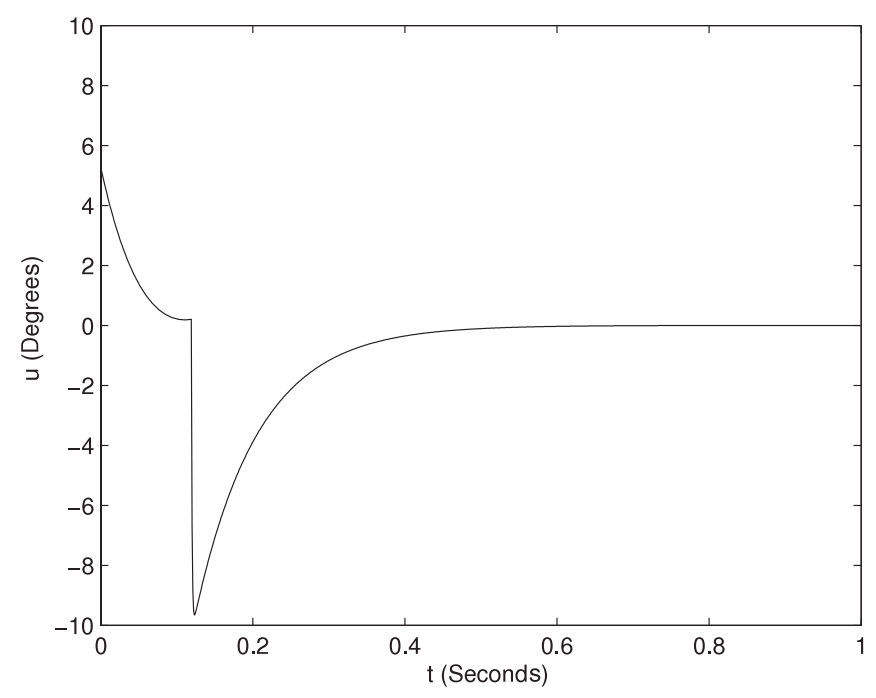

FIGURE 3. The deflection angle of the elevator under the single sliding mode controller law.

where $S_{i}(\boldsymbol{x}) \neq 0$. Thus, it follows from the Lyapunov stability theory that the system is asymptotically stable to the sliding surface $S(x)=0$.

Combining the stability of the system in the sliding mode phase and in the reaching phase, we conclude that the system is asymptotically stable in the whole state space. This completes the proof.

\section{Simulation}

For certain cruise missiles, they fly at a specific height. Suppose that the maximal allowable values on the deflection angle of the elevator is \pm 6 degrees. Since the flight height is high, the deflection angle of the elevator will exceed these maximal values if a single sliding surface control law is used to track the overload signal. The curve showing the deflection angle of the elevator for the single sliding surface is depicted in Figure 3. We can see that it exceeds the maximal allowable values. In order to overcome this problem, we design a multiple sliding mode control law. The curve showing the deflection angle of the elevator under the multiple sliding mode controller is as shown in Figure 4. From this simulation curve, we see that the elevator is operated within the engineering specification. The state trajectory under the multiple sliding mode control law is as shown in Figure 5. The tracking of the system output to the unit overload is depicted in Figure 6.

In the simulation study the parameters are as follows: $T_{M}$, the time constant of the missile, is $0.1427 ; \xi_{M}$, the relative damping coefficient of the missile, is $0.049 ; K_{M}$, the transfer coefficient of the missile, is -0.128 ; $v$, the flight speed of the missile, is $3 \mathrm{Ma} ; g$, the gravitational acceleration, is $9.8 ; C_{s}$, the target sliding mode parameter, 


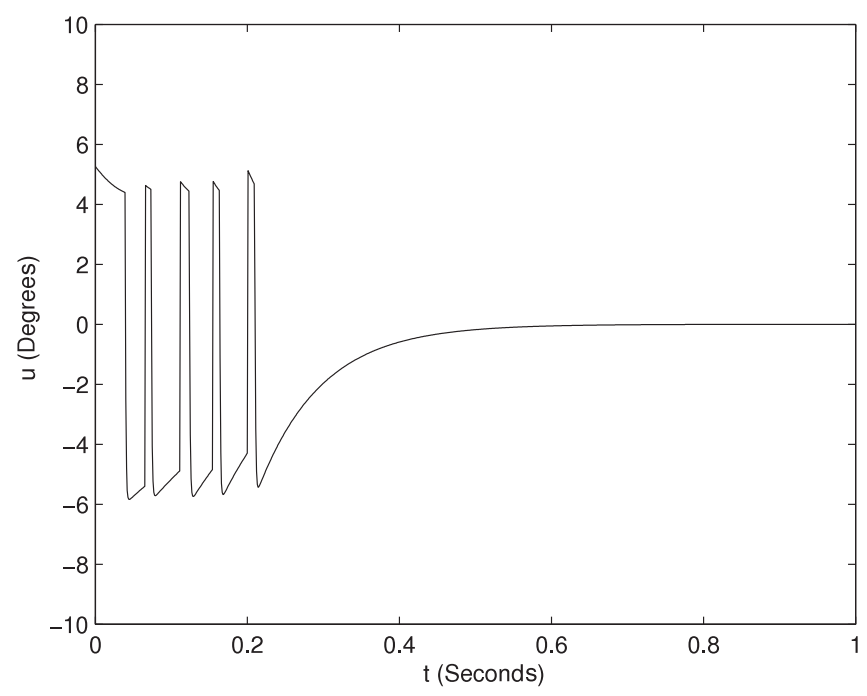

FIGURE 4. The deflection angle of the elevator under the multiple sliding mode controller law.

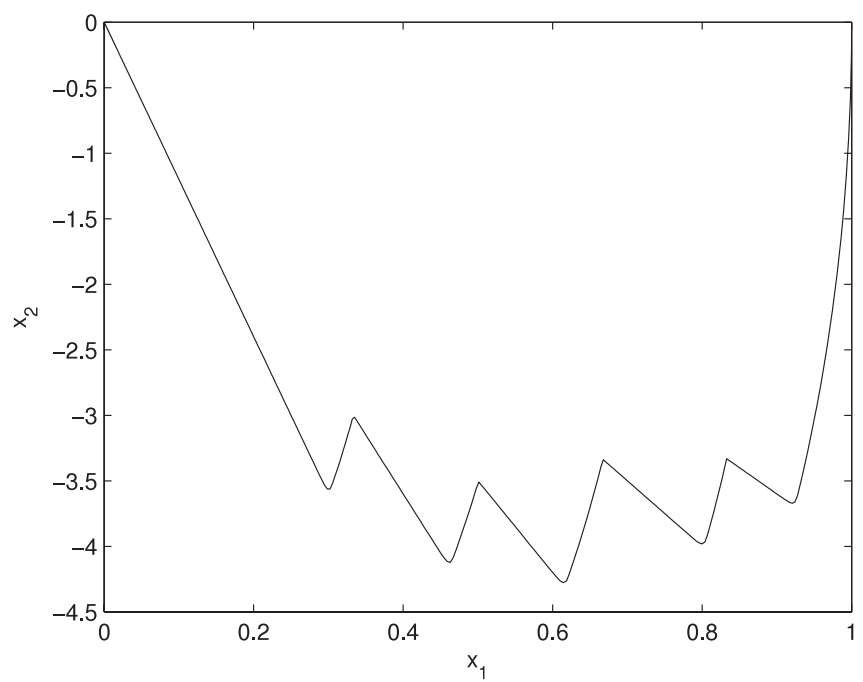

FIGURE 5. The phase plane plot under the multiple sliding mode control law.

is chosen as $12 ; C_{r}$, the reaching parameter, is $100 ; \delta$, the chattering elimination parameter, is 0.1 ; and the simulating step is taken to be $0.001 \mathrm{~s}$.

\section{Conclusions}

In this paper, a design method for a linear multiple sliding mode recursive control law is proposed. The Lyapunov stability theory was used to establish the stability of 


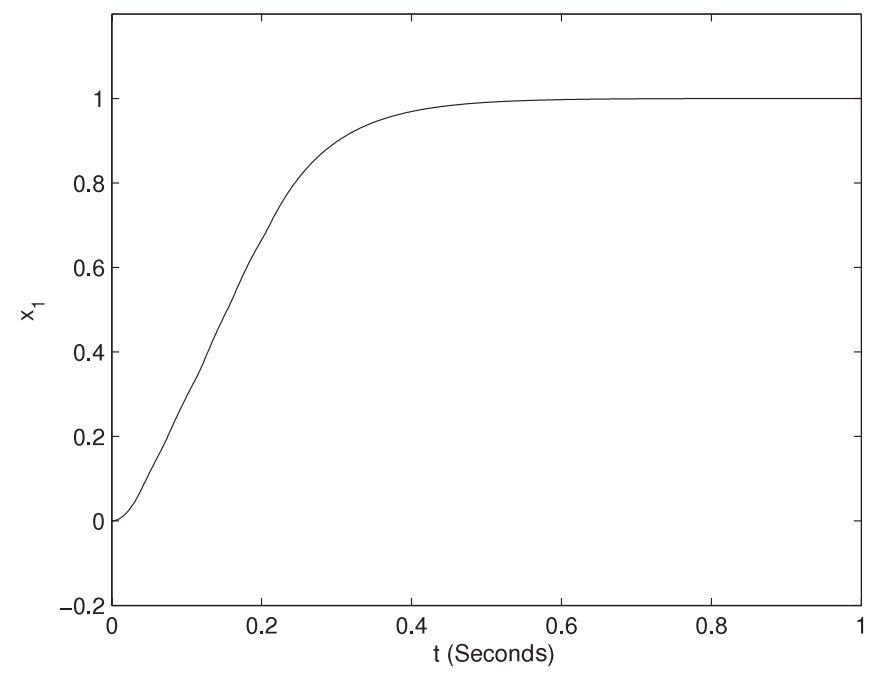

FIGURE 6. The tracking of the system output to the unit overload.

the system. To eliminate the chattering of the system, a simple saturation function method is used. The design method is practical and easy to implement. Furthermore, it ensures that the control obtained will operate within the engineering specification.

\section{Acknowledgements}

This work was supported by a grant from the National Natural Science Foundation of China (60774062), and a grant from the Education University Doctor Foundation of Chinese Ministry (20050213010).

\section{References}

[1] Y. A. Hu and Y. Chen, "Design and application of double-sliding modes variable structure control systems", in Proc. Fifth Int. Symp. on Instrumentation and Control Technology (Proceedings of the SPIE, Beijing, China, 2003) 635-638.

[2] X. H. Liao, Z. Sun and Y. D. Song, "Chattering-free variable structure control with application to flight vehicles", in Proc. 38th Southeastern Symp. on System Theory (Tennessee Technological University, Cookeville, TN, 2006) 406-410.

[3] A. R. Mehrabian and J. Roshanian, "Skid-to-turn missile autopilot design using scheduled eigenstructure assignment technique”, Proc. Inst. Mech. Eng. G, J. Aerospace Eng. 220 (2006) 225-239.

[4] Y. Pan, K. Furuta, S. Suzuki and S. Hatakeyama, "Design of variable structure controller-from sliding mode to sliding sector", in Proc. IEEE Conf. on Decision and Control (IEEE Computer Society, Sydney, Australia, 2000) 1685-1690.

[5] M. U. Salamci, M. K. Ozgoren and S. P. Banks, "Sliding mode control with optimal sliding surfaces for missile autopilot design”, J. Guid. Control Dyn. 23 (2000) 719-727.

[6] T. Shima, M. Idan and O. M. Golan, "Sliding-mode control for integrated missile autopilot guidance", J. Guid. Control Dyn. 29 (2006) 250-260. 
[7] Y. J. Shi, G. F. Ma and H. Z. Ma, "Study of the compound control of cruise missile based on variable structure control method", J. Ballistics, in press, 2008.

[8] Y. B. Shtessel, I. A. Shkolnikov and A. Levant, "Smooth second-order sliding modes: missile guidance application", Automatica 43 (2007) 1470-1476.

[9] K. K. Shyu and C. Y. Lin, "Adaptive sliding mode control for variable structure systems with constraint control input", Dynam. Control 6 (1996) 49-61.

[10] J. H. Wei and C. Guan, "Derivation and integral sliding mode variable structure control of hydraulic velocity tracking system", Chin. J. Mech. Eng. 18 (2005) 224-227.

[11] Y. S. Zhao, Y. Q. Zhang, J. Yang and L. P. Chen, "Enhanced fuzzy sliding mode controller for robotic manipulators", Int. J. Robot. Autom. 22 (2007) 170-183.

[12] D. Zhou, C. D. Mu and W. L. Xu, "Adaptive sliding-mode guidance of a homing missile", J. Guid. Control Dyn. 22 (1999) 589-594. 